\title{
Reduction of Equivalent Series Inductor Effect in Delay-Ripple Reshaped Constant On-Time Control for Buck Converter With Multilayer Ceramic Capacitors
}

\author{
Wei-Chung Chen, Student Member, IEEE, Ching-Sung Wang, Yi-Ping Su, Yu-Huei Lee, Student Member, IEEE, \\ Chia-Ching Lin, Ke-Horng Chen, Senior Member, IEEE, and Ming-Jhe Du
}

\begin{abstract}
The stability of conventional constant on-time control buck converter is constrained by the time constant, which is the product of the output capacitor and its equivalent series resistance (ESR). Specialty polymer capacitor which is mostly used as output capacitor for such a consideration although it limits the performance of converter. On the other hand, the multilayer ceramic capacitors are widely used in commercial power management ICs due to the advantages of low cost and ESR. However, the stability often confronts with the subharmonic problem caused by small time constant. A differential-zero compensator with the noise margin enhancement (DZC-NME) technique in constant on-time control buck dc-dc converter with output ceramic capacitor is proposed in this paper. Thus, the proposed DZC-NME technique not only eliminates the limit of large time constant but also tolerates the existence of equivalent series inductor (ESL) effect. Experiment results demonstrate small output ripple of $10 \mathrm{mV}$ and high efficiency of 91\% when ESR is smaller than $1 \mathrm{~m} \Omega$ and large interference from ESL effect is $40 \mathrm{mV}$.
\end{abstract}

Index Terms-Constant on-time control for buck converter, equivalent series inductor (ESL), multilayer ceramic capacitors (MLCC), power management.

\section{INTRODUCTION}

$\mathbf{P}$ OWER management module plays an important role in portable products to extend the battery life and provide the high-quality power supply [1]-[4]. There are various kinds of topology for meeting different required specifications and applications [5]-[11]. As a result, the constant on-time (COT) control has been widely used due to its high efficiency, good transient response, and simple control mechanism [12], [13]. As illustrated in Fig. 1, conventional COT control in the buck

Manuscript received March 9, 2012; revised June 5, 2012; accepted August 18, 2012. Date of current version November 22, 2012. This work was supported by the Bureau of Energy. Recommended for publication by Associate Editor $\mathrm{H}$. S. H. Chung.

W.-C. Chen, Y.-P. Su, Y.-H. Lee, C.-C. Lin, and K.-H. Chen are with the Institute of Electrical and Computer Engineering, National Chiao Tung University, Hsinchu 300, Taiwan (e-mail: weichung330@ gmail. com; susu.supy@gmail.com; leeyuhuei@gmail.com; suimono@gmail.com; khchen@cn.nctu.edu.tw).

C.-S. Wang is with the Department of Electronic Engineering, Oriental Institute of Technology, Taipei 22061, Taiwan (e-mail: ff020@ mail.oit.edu.tw).

M.-J. Du is with the Industrial Technology Research Institute, Hsinchu 31040, Taiwan (e-mail: mjdu@itri.org.tw).

Color versions of one or more of the figures in this paper are available online at http://ieeexplore.ieee.org.

Digital Object Identifier 10.1109/TPEL.2012.2215886

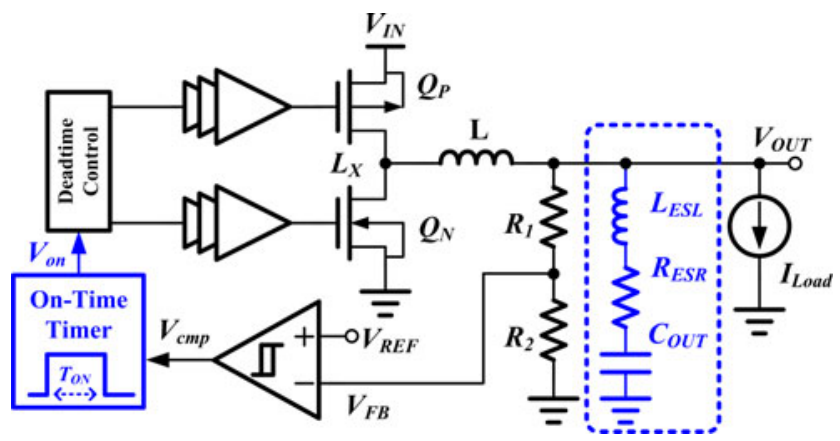

Fig. 1. Architecture of the COT control in conventional dc-dc buck converter.
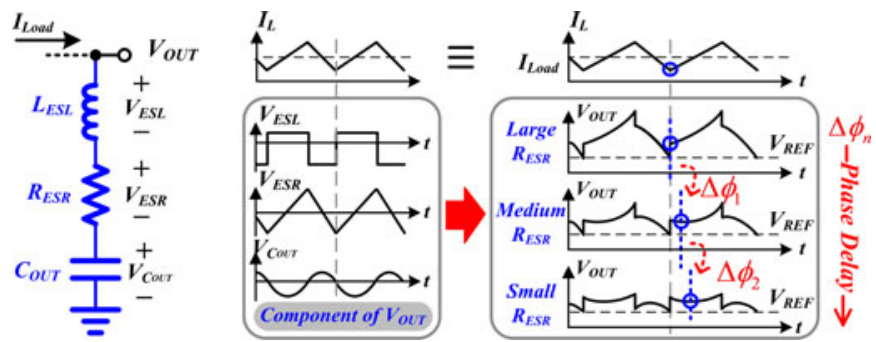

Fig. 2. Relationship between the output voltage ripple and the inductor current under different $R_{\mathrm{ESR}}$.

converter is constructed by comparator, on-time timer, MOSFETs as switches, and the energy storage components, inductor, and capacitors. Once the feedback voltage $V_{\mathrm{FB}}$ falls below the reference voltage $V_{\mathrm{REF}}$ and is detected by the comparator, the one-shot timer is triggered to increase the inductor current until a predefined on-time $T_{\mathrm{ON}}$ expires. To ensure system stability, the inductor current information derived from the output ripple is used as the ramp signal in the pulsewidth modulation (PWM) to determine duty cycle. Basically, in Fig. 2, the output voltage ripple caused by the inductor current ripple contains three major terms, which are $V_{\mathrm{ESL}}, V_{\mathrm{ESR}}$, and $V_{\mathrm{COUT}}$ contributed by the parasitic effect on the equivalent series inductance (ESL), the equivalent series resistance (ESR), and the output capacitor $C_{\mathrm{OUT}}$, respectively.

Under the same output capacitor value, different ESR value $R_{\mathrm{ESR}}$ results in different output ripple. It can be translated to the phase delay $\Delta \Phi$ related to the inductor current $I_{L}$ owing to variable $R_{\mathrm{ESR}}$. The smaller the $R_{\mathrm{ESR}}$, the longer phase 
TABLE I

SORTS OF CAPACITOR

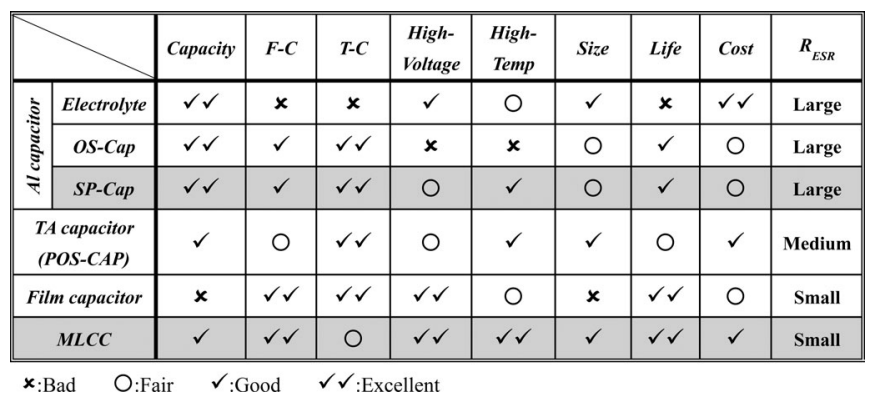

delay is observed at the lowest point of the $V_{\mathrm{OUT}}$. It will worsen the system stability. In other words, the switching frequency should be kept high enough to overcome the phase delay for system stability. However, it is not practical to raise the switching frequency to higher than megahertz because the switching loss degrades the efficiency. The phase delay is basically caused by the type of the output capacitor. That is, the selection of the output capacitor will seriously affect the system stability if the COT control is used.

Table I shows various kinds of capacitor including electrolyte capacitor, OS-Cap, SP-Cap, POS-CAP, film capacitor, and multilayer ceramic capacitor (MLCC), and ranks their performance according to each characteristic. Here, $F-C$ and $T-C$ represent its variation of quality at different frequency and temperature, respectively. High-Voltage and High-Temp represent the limit of highest voltage and temperature, respectively. Especially, the SP-Cap is the most common choice in applications with conventional COT control buck converter since the system stability is guaranteed only with large ESR value, but the output ripple is large. The inexpensive MLCC will be excluded due to its low ESR. However, the MLCC is one of the suitable choices owing to it low cost and other superior characteristics as listed in Table I [14], [15]. Therefore, to ensure system stability and low cost, the differential-zero-compensator with the noise margin enhancement (DZC-NME) technique is proposed. In other words, the system stability is increased by the DZC-NME technique without being affected by phase delay when the MLCC is utilized.

In this paper, the structure of conventional COT control buck converter is introduced in Section II to reveal the stability criteria. Then, the proposed COT control with the DZC-NME technique is described in Section III to show the high performance with the MLCC. The circuit implementation is presented in Section IV. Experimental results are shown in Section V. Finally, the conclusion is made in Section VI.

\section{Disadvantage of Conventional Adaptive COT CONTROL In Buck CONVERTER STRUCTURE}

The derivation of the stability criteria of the adaptive COT control can reveal the idea of the DZC-NME technique to guarantee the system stability with the use of the MLCC.

The COT control does not have an internal clock in the whole system. Thus, the COT control should still keep a constant

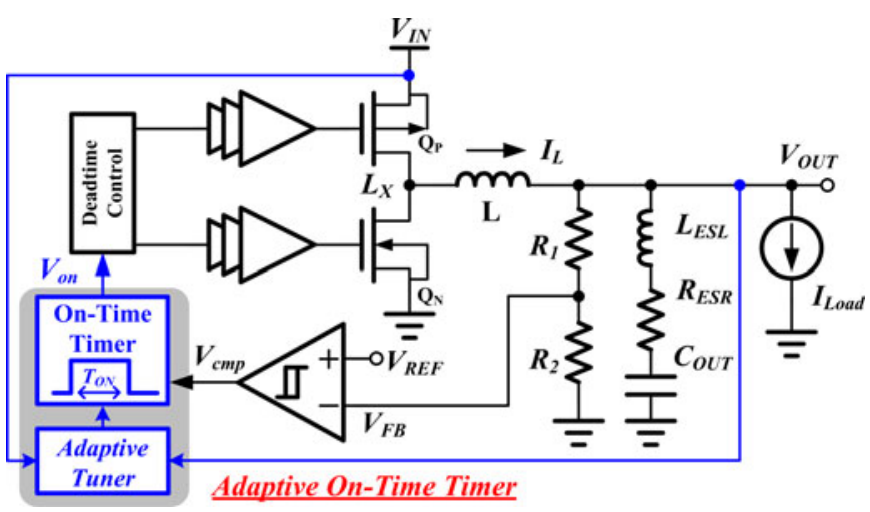

Fig. 3. DC-DC buck converter with the adaptive on-time control.

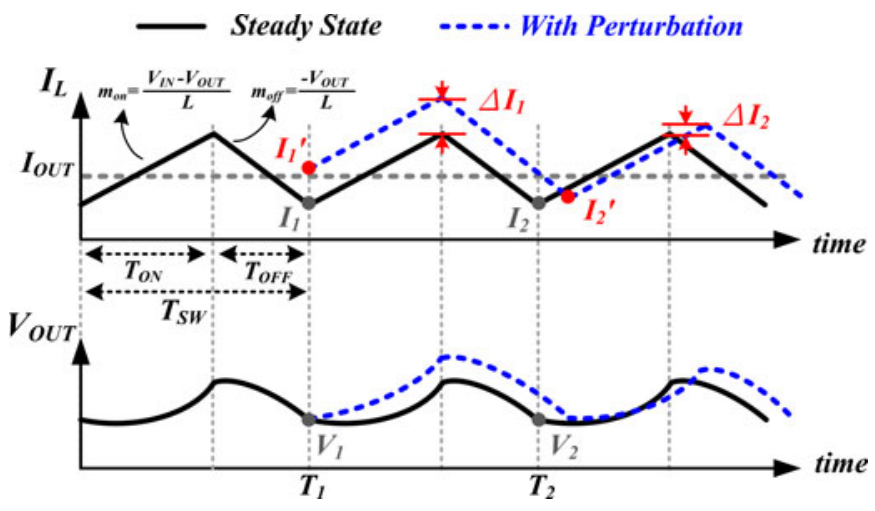

Fig. 4. Steady-state waveforms and with the insertion of the perturbation.

switching frequency at the continuous conduction mode (CCM) operation to ensure that the electromagnetic interference (EMI) can be minimized by a specific output filter. For maintaining the switching frequency constant, the adaptive on-time circuit adjusts the on-time period according to the input voltage $V_{\text {IN }}$ and the output voltage $V_{\mathrm{OUT}}$, as shown in Fig. 3 . As a result, the switching frequency is determined by (1) [16]. If the on-time $T_{\mathrm{ON}}$, as expressed in (2), is inversely proportional to the $V_{\mathrm{IN}}$ but directly proportional to the $V_{\mathrm{OUT}}$, the switching frequency $f_{S W}$ will be nearly constant as shown in (3)

$$
\begin{aligned}
f_{\mathrm{SW}} & =\frac{V_{\mathrm{OUT}}}{V_{\mathrm{IN}} T_{\mathrm{ON}}} \\
T_{\mathrm{ON}} & =\frac{V_{\mathrm{OUT}}}{V_{\mathrm{IN}}} \times k_{1}, \quad \text { where } k_{1}=\text { constant } \\
f_{\mathrm{SW}} & =\frac{V_{\mathrm{OUT}}}{V_{\mathrm{IN}} \cdot\left(\left(V_{\mathrm{OUT}} / V_{\mathrm{IN}}\right) \cdot k_{1}\right)}=k_{1} .
\end{aligned}
$$

According to the steady-state waveforms in Fig. 4 if the ESL effect is ignored, the voltage difference should be zero after one switching period as expressed in (4). The switching period $T_{S W}$ is the summation of the $T_{\mathrm{ON}}$ and the $T_{\mathrm{OFF}}$, which are the on-time and the off-time, respectively

$$
\begin{aligned}
V_{2}-V_{1}= & \frac{1}{C_{\mathrm{OUT}}} \int_{0}^{T_{\mathrm{SW}}}\left(I_{L}(t)-I_{\mathrm{OUT}}\right) d t \\
& +\left(I_{2}-I_{1}\right) R_{\mathrm{ESR}}=0
\end{aligned}
$$


where

$$
\begin{aligned}
& T_{\mathrm{SW}}=T_{\mathrm{ON}}+T_{\mathrm{OFF}} \quad \text { and } \\
& \begin{cases}I_{L}(t)=\frac{V_{\mathrm{IN}}-V_{\mathrm{OUT}}}{L} t, & \text { in on-time period } \\
I_{L}(t)=\frac{-V_{\mathrm{OUT}}}{L} t, & \text { in off-time period }\end{cases}
\end{aligned}
$$

Besides, the $T_{\mathrm{OFF}}$ can be expressed as

$$
T_{\mathrm{OFF}}=\left(\frac{V_{\mathrm{IN}}}{V_{\mathrm{OUT}}}-1\right) T_{\mathrm{ON}}
$$

Substituting (5) into (4), following equation can be derived after evaluating integers and simplifying

$$
\begin{aligned}
& \frac{V_{\mathrm{IN}}}{V_{\mathrm{OUT}}} \frac{T_{\mathrm{ON}}}{C_{\mathrm{OUT}}}\left(I_{1}-I_{\mathrm{OUT}}\right)+\frac{T_{\mathrm{ON}}^{2}}{2 C_{\mathrm{OUT}} L_{1}} \frac{V_{\mathrm{IN}}}{V_{\mathrm{OUT}}}\left(V_{\mathrm{IN}}-V_{\mathrm{OUT}}\right) \\
& \quad+R_{\mathrm{ESR}}\left(I_{2}-I_{1}\right)=0 .
\end{aligned}
$$

To conclude the stability criteria and linearize the (6), the small perturbation signals $\Delta I_{1}$ and $\Delta I_{2}$ are taken into consideration in the steady-state inductor currents $I_{1}$ and $I_{2}$, which are shown in (7), at the time of $T_{1}$ and at time of $T_{2}$, respectively, according to the inductor current waveforms in Fig. 4

$$
\left\{\begin{array}{l}
I_{1}=I_{\mathrm{OUT}}-\frac{T_{\mathrm{ON}}}{2 L}\left(V_{\mathrm{IN}}-V_{\mathrm{OUT}}\right) \\
I_{2}=I_{\mathrm{OUT}}-\frac{T_{\mathrm{ON}}}{2 L}\left(V_{\mathrm{IN}}-V_{\mathrm{OUT}}\right) .
\end{array}\right.
$$

Consequently, the inductor currents $I_{1}^{\prime}$ and $I_{2}^{\prime}$ with perturbation at the time of $T_{1}$ and at the time of $T_{2}$, respectively, can be expressed as

$$
\left\{\begin{array}{c}
I_{1}^{\prime}=\Delta I_{1}+I_{\mathrm{OUT}}-\frac{T_{\mathrm{ON}}}{2 L}\left(V_{\mathrm{IN}}-V_{\mathrm{OUT}}\right) \\
I_{2}^{\prime}=\Delta I_{2}+I_{\mathrm{OUT}}-\frac{T_{\mathrm{ON}}}{2 L}\left(V_{\mathrm{IN}}-V_{\mathrm{OUT}}\right) \\
I_{2}^{\prime}-I_{1}^{\prime}=\Delta I_{2}-\Delta I_{1} .
\end{array}\right.
$$

Substituting (8) into (6), (9) can be derived as

$$
\Delta I_{1}\left(R_{\mathrm{ESR}}-\frac{V_{\mathrm{IN}}}{V_{\mathrm{OUT}}} \frac{T_{\mathrm{ON}}}{C_{\mathrm{OUT}}}\right)-\Delta I_{2} R_{\mathrm{ESR}}=0 .
$$

For increasing stability, $\Delta I_{2} / \Delta I_{1}$ must be gradually converged to zero in steady state. Thus, the inequality can be derived as (10) and simplified as (11)

$$
\begin{aligned}
\left|\frac{\Delta I_{2}}{\Delta I_{1}}\right| & =\left|\frac{R_{\mathrm{ESR}}-\left(V_{\mathrm{IN}} / V_{\mathrm{OUT}}\right)\left(T_{\mathrm{ON}} / C_{\mathrm{OUT}}\right)}{R_{\mathrm{ESR}}}\right|<1 \\
0 & <\frac{1}{R_{\mathrm{ESR}} C_{\mathrm{OUT}}}<2 \frac{V_{\mathrm{OUT}}}{V_{\mathrm{IN}} T_{\mathrm{ON}}} .
\end{aligned}
$$

Finally, (12) is obtained to indicate that the stability is related to the relationship between the $R_{\mathrm{ESR}}$ and the $C_{\mathrm{OUT}}$ under the consideration of the switching frequency

$$
\begin{aligned}
f_{\mathrm{SW}} & >\pi f_{\mathrm{ESR}}, \quad \text { where } f_{\mathrm{SW}}=\frac{V_{\mathrm{OUT}}}{V_{\mathrm{IN}} T_{\mathrm{ON}}}, \\
f_{\mathrm{ESR}} & =\frac{1}{2 \pi \cdot R_{\mathrm{ESR}} C_{\mathrm{OUT}}} .
\end{aligned}
$$

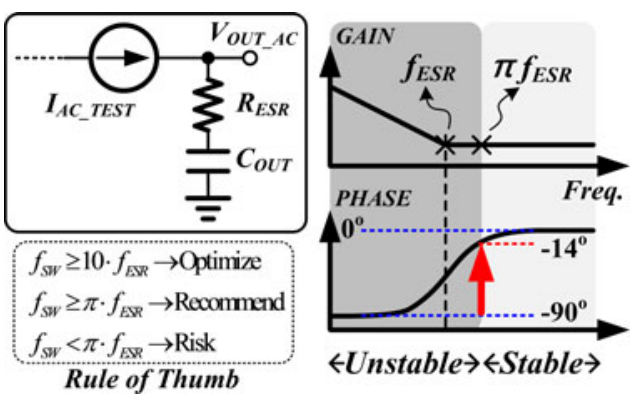

Fig. 5. Test model of the output capacitor and its frequency response with the stability criteria.

Obviously, due to this limitation, the specific material of capacitor is necessary to guarantee the system stability. Certainly, this constraint derived in this paper is more rigorous than that in the prior arts [13]. Furthermore, (12) can also correspond to the frequency response as shown in Fig. 5. The obtained ac response of output $V_{\text {OUT_AC }}$ indicates the design guideline of the COT control. The $R_{\mathrm{ESR}}$ and the $C_{\mathrm{OUT}}$ contribute a zero to reduce the phase delay between the inductor current ripple and output voltage ripple. That is, the system stability can be guaranteed since the phase is boosted from $-90^{\circ}$ to $-14^{\circ}$ at the desired operation switching frequency. Consequently, for adequate in-phase relationship between the two signals, it necessary to make sure the switching frequency is high enough.

The specialty polymer capacitor (SP-CAP) with a value of $200 \mu \mathrm{F}$ has the ESR in tens of milliohms. It means that the $f_{\mathrm{ESR}}$ is near $50 \mathrm{kHz}$. That is, the $f_{S W}$ needs to be great than $157 \mathrm{kHz}$ for the maximum phase delay of $14^{\circ}$. It is easy to find some suitable SP-CAPs to increase the stability of the COT control system but the cost is high and the magnitude of output ripple is large. On the other hand, the ESR value of MLCC is only several milliohms at the same output capacitor value. It pushes the lowest allowable switching frequency to $1.4 \mathrm{MHz}$ or even higher than it. However, higher switching operation will cause large switching loss and worse jitter problem. It results that the MLCC is not suitable for the conventional adaptive COT control.

Thus, the DZC-NME technique is proposed to compensate the delay of phase caused by the MLCC for increasing stability with low cost.

\section{Proposed COT Control Buck Structure AND SYSTEM LOOP ANALYSIS}

In the COT-control buck converter with low-ESR output capacitor, the low-ripple $V_{\mathrm{OUT}}$ is fed back to the controller by the voltage divider. Thus, the voltage ripple at the phase-delay $V_{\mathrm{FB}}$, which is compared to the $V_{\mathrm{OUT}}$, is smaller and has low noise immunity. Fig. 6 is the architecture of COT-control dc-dc buck converter and shows the proposed differential-zero compensator with the noise margin enhancement circuit (DZC-NME), which is composed of the differential-zero compensator (DZC) technology and the noise margin enhancement circuit (NME).

The $V_{\mathrm{cmp}}$ triggers the adaptive on-time timer (AONT) circuit to generate a COT period and thus the inductor current increases. 


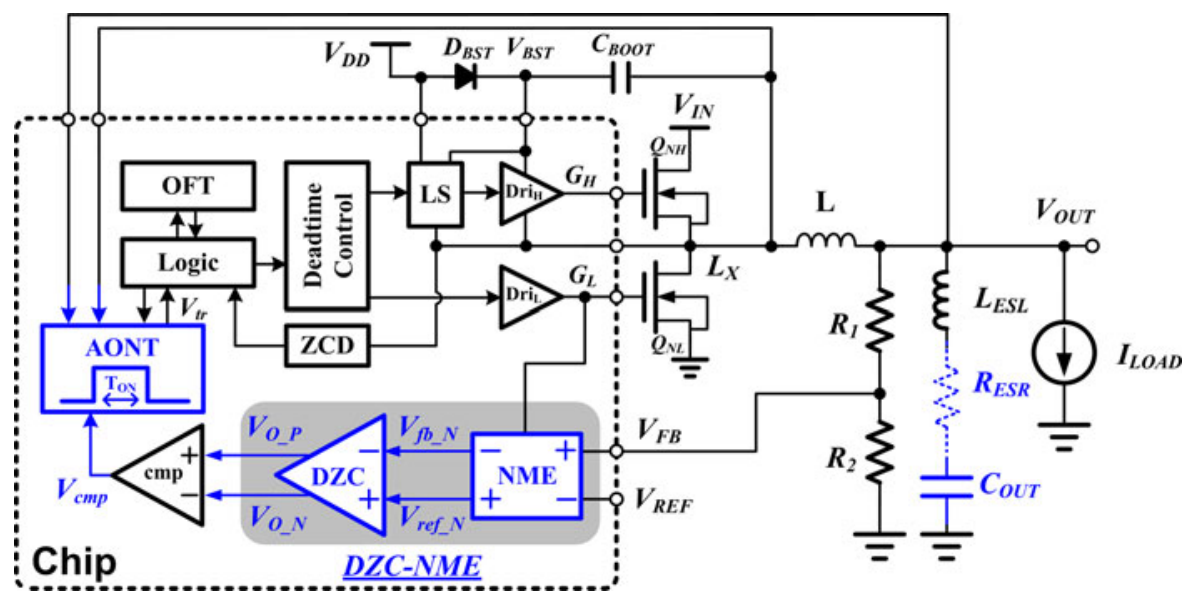

Fig. 6. Architecture of COT-control dc-dc bootstrap buck converter with the proposed DZC-NME technique.

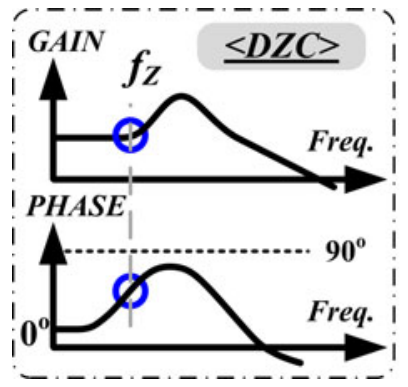

Fig. 7. Phase-lead effect caused by the proposed DZC technique can increase the system stability.

On the other hand, after the end of the on-time, the inductor current decreases until the $V_{\mathrm{FB}}$ falls below the reference voltage, $V_{\mathrm{REF}}$, or the end time of minimum off-time generated by the offtime timer circuit. The minimum off-time is necessary to avoid over current or overshoot of output voltage caused by extreme duty condition during the start-up period or in case of light-toheavy load variation. Besides, the zero-current detector (ZCD) circuit offers the function of discontinuous conduction mode (DCM) to further reduce the switching frequency for enhancing the power conversion efficiency at light loads.

When using MLCC as output capacitor with a low ESR, the output ripple voltage is mainly determined by the ESL and the charging/discharging on output capacitor $C_{\mathrm{OUT}}$. The low value of $R_{\mathrm{ESR}}$ which generates a high-frequency zero offers little assistance to increase phase margin. The instability caused by the low ESR value can be compensated by the DZC circuit, which corresponds to the proportional-differential (PD) controller with the frequency response as illustrated in Fig. 7. That is, the DZC circuit boosts the phase to meet the limited condition as 14 degrees at required frequency about $300 \mathrm{kHz}$ and releases the conventional restriction without the need of large $R_{\mathrm{ESR}}$ and $C_{\text {OUT }}$.

However, the existing ESL ( $L_{\mathrm{ESL}}$ in Fig. 6) distorts the feedback control signal $V_{\mathrm{FB}}$ by the voltage step, $V_{\mathrm{ESL}}$, as shown in (13) and (14). It results that the on-time timer might be triggered at the incorrect time. As depicted in Fig. 8, the $V_{\mathrm{ESL}}$ is proportional to the $V_{\mathrm{IN}}$ and the $L_{\mathrm{ESL}}$. The $V_{\mathrm{ESL}}$ is as large as $42 \mathrm{mV}$

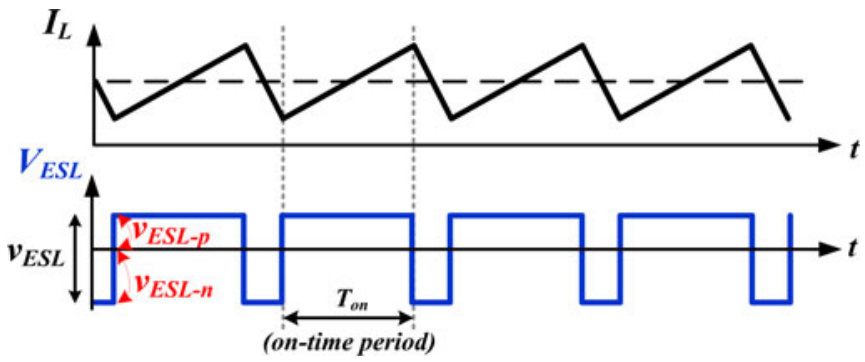

Fig. 8. ESL effect at the output voltage.

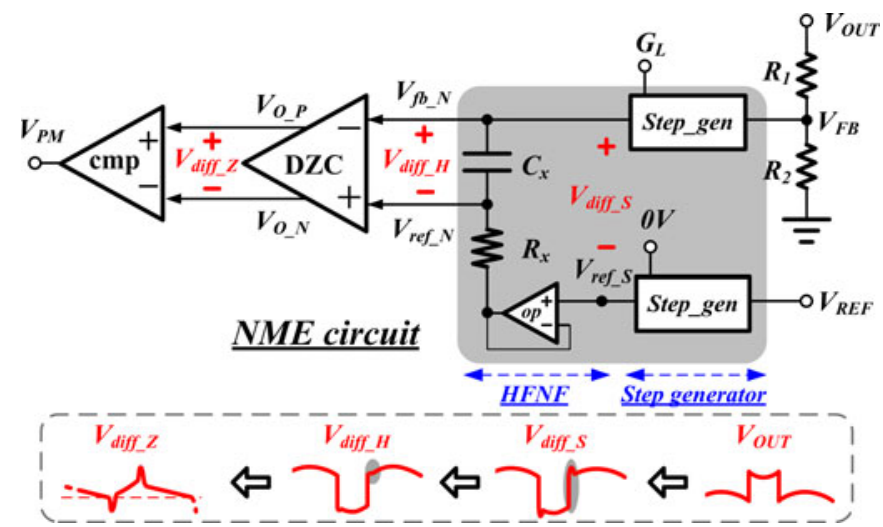

Fig. 9. Structure of the NME circuit.

when the $V_{\mathrm{IN}}$ is $21 \mathrm{~V}$ and the $L_{\mathrm{ESL}}$ is $1 \mathrm{nH}$ if $L$ is $2 \mu \mathrm{H}$

$$
v_{\mathrm{ESL}}=v_{\mathrm{ESL}-p}+\left|v_{\mathrm{ESL}-n}\right|=\frac{V_{\mathrm{IN}}}{L} \cdot L_{\mathrm{ESL}}
$$

where

$v_{\mathrm{ESL}-p}=\frac{V_{\mathrm{IN}}-V_{\mathrm{OUT}}}{L} \cdot L_{\mathrm{ESL}}$ and $v_{\mathrm{ESL}-n}=\frac{-V_{\mathrm{OUT}}}{L} \cdot L_{\mathrm{ESL}}$.

Therefore, the NME circuit as illustrated in Fig. 9 is proposed to alleviate the ESL effect for higher noise margin [13]. Here, the differential outputs of the step generator, the high-frequency 


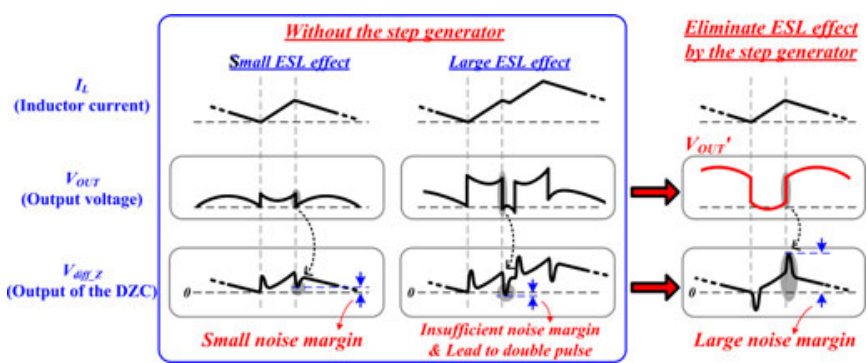

Fig. 10. Function of the step generator.

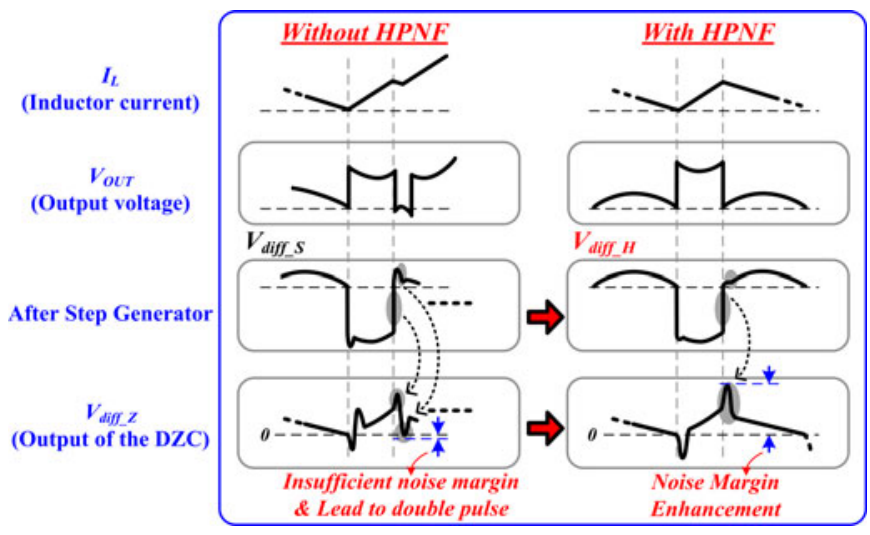

Fig. 11. Waveforms for illustrating the function of the DZC-NME technique.

noise filter (HFNF), and the DZC are the $V_{\text {diff_S }}$, the $V_{\text {diff_H }}$, and the $V_{\text {diff_Z }}$, respectively.

The $V_{\mathrm{ESL}}$, one of the components of the $V_{\mathrm{OUT}}$, is a step function. In Fig. 10 in case of a small ESL effect, the downstepping $V_{\mathrm{ESL}}$ at the beginning of off-time transfers to undershoot of the differential signal $V_{\text {diff_Z }}$ after the process of the DZC circuit but without the step generator circuit. Undershoot may drop below the $0 \mathrm{~V}$ caused by the ESL effect and incorrect triggering effect at the beginning of off-time instantly decreases the system stability. In other words, phenomenon of double pulse happens. To alleviate the unstable phenomenon, the proposed step generator creates an opposite step and thus the $V_{\mathrm{OUT}}{ }^{\prime}$ is revised from the $V_{\text {OUT }}$. As a result, overshoot of the $V_{\text {diff }} Z$ at the beginning of off-time greatly enhances the noise margin. The $V_{\text {diff_Z }}$ is reshaped and is in phase with the inductor current after the overshoot. On the other hand, there is no need to take concern about the undershoot of the $V_{\mathrm{diff} \_Z}$, which is distorted drastically in the beginning of on-time, because the on-time is innately defined in the AONT circuit.

In order to eliminate the effect of the $V_{\mathrm{ESL}}$, the opposite step must be generated and synchronized with the period of on-time. However, there is a limitation in bandwidth and phase margin (PM) for the step generator. If sacrificing PM to obtain enough speed, the differential output of the step generator $V_{\text {diff_S }}$ as shown in Fig. 11 will lead to double pulse after the DZC. For covering the defect of the step generator, the high-frequency noise filter circuit offers a solution to relieve it by coupling the high-frequency variation from one input terminal to the other terminal of the DZC. Consequently, the $V_{\mathrm{FB}}$ is preregulated by the NME circuit to enhance noise immunity at first, and then

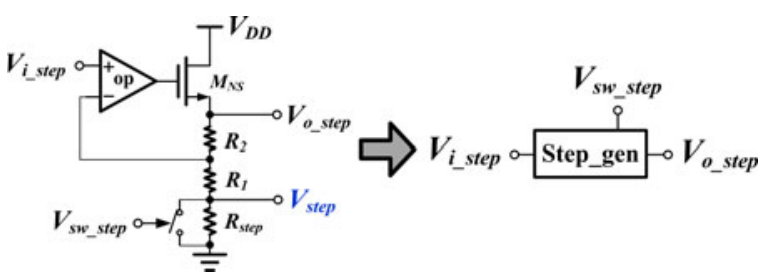

Fig. 12. Structure of the step generator.

regulated by the DZC technique. The differential output signal of the DZC, $V_{\text {diff } Z Z}$, is reshaped from the phase-delay $V_{\mathrm{FB}}$ and thus in phase with the inductor current during the off-time period.

\section{CiRCUIT IMPLEMENTATION}

The proposed DZC-NME technique is implemented in the $\mathrm{dc}-\mathrm{dc}$ buck converter with the bootstrap architecture as depicted in Fig. 6. It is mainly composed of two $N$-channel power MOSFETs $\left(Q_{N H}\right.$ and $\left.Q_{N L}\right)$ working as high-side and low-side switches. For completely turning $\mathrm{ON}$ the high-side switch, the level-shift circuit (LS) is used to level up the driving signal to the value of " $V_{\mathrm{DD}}+\mathrm{V}_{\mathrm{IN}}-V_{D}$ ". The $V_{\mathrm{DD}}$ and the $V_{\mathrm{IN}}$ are supply voltages of the chip and the converting source voltage, respectively. The $V_{D}$ is drop voltage of bootstrapping diode $D_{\mathrm{BST}}$. Within the off-time period, the $C_{B O O T}$ is charged to $V_{\mathrm{DD}}-$ $\mathrm{V}_{D}$. The $V_{\mathrm{IN}}$ is pumped up to the value of " $V_{\mathrm{DD}}+\mathrm{V}_{\mathrm{IN}}-V_{D}$ " when the high-side power MOSFET turns ON.

\section{A. DZC-NME Circuit}

To analyze the function of the proposed DZC-NME technique, the DZC-NME technique is divided into two parts, the DZC and the NME. The NME as shown in Fig. 9 is composed of the step generator and the HFNF. The HFNF consists of the $C_{X}$, the $R_{X}$ and a buffer which is to block the interference to the $V_{\mathrm{REF}}$. The HFNF is used for coupling the same magnitude of instantaneous variation from the $V_{\mathrm{FB}}$, such as the step of the $V_{\mathrm{ESL}}$ as shown in Fig. 2 or any high-frequency noise, to both two input terminals of the DZC.

The step generator is implemented as shown in Fig. 12. With the $M_{N S}$, the op-amp and the resistor divider, this structure forms as a negative feedback to decide the output signal, $V_{o_{-} \text {step }}$, linearly depending on the input signal, $V_{i_{-} \text {step }}$. The switch across the $R_{\text {step }}$ is triggered by the control signal $G_{L}$ of the low-side power MOSFET and the $V_{o \_ \text {step }}$ goes toward high or low with the switch turning ON or OFF, respectively. The step magnitude can be derived as (15). Relative to the $V_{\mathrm{ESL}}$, this opposite step enhances noise margin and avoids incorrect trigger caused by the step of the $V_{\mathrm{ESL}}$

$$
\Delta V_{\text {o_step }}=\frac{R_{2} R_{\text {step }}}{R_{1}\left(R_{1}+R_{\text {step }}\right)} V_{i_{-s t e p}}
$$

The differential zero compensator circuit is depicted as shown in Fig. 13. The $P_{1}$ and the $P_{2}$ constitute the input differential pair. The $N_{1}$ and the $N_{2}$ form active load. $R_{1}, C_{1}, R_{2}$, and $C_{2}$, are used to create low-frequency zero for the compensation. In 


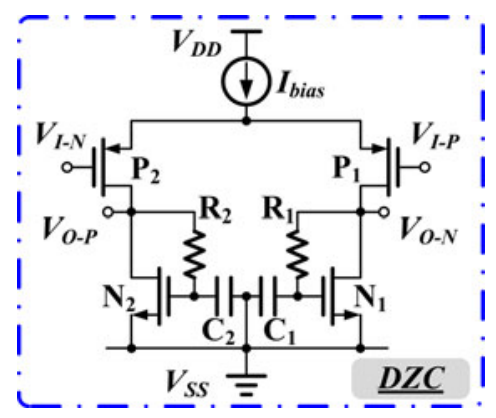

Fig. 13. Implementation of the DZC.

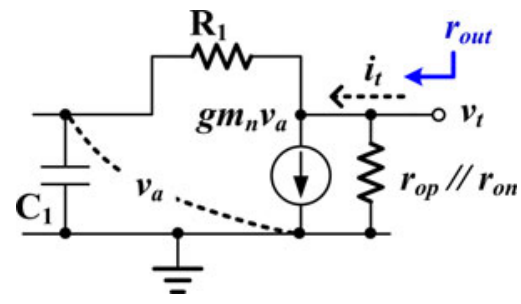

Fig. 14. Small-signal model of the DZC circuit.

the meanwhile, low-output impedance, which is generated by the pseudo-diode connection of the $N_{1}$ and the $N_{2}$, will result in high-frequency pole and lead to the function of phase lead of this compensator.

Fig. 14 indicates the corresponding small signal model of the DZC circuit. Setting the test voltage source $v_{t}$ at the output of the DZC circuit, the output current $i_{t}$ flows into the circuit.

By the KCL theorem, the $i_{t}$ is expressed as

$$
i_{t}=\frac{v_{t}}{r_{\mathrm{on}} / / r_{\mathrm{op}}}+\frac{v_{t}}{R+(1 / s C)}+g m \cdot v_{a} .
$$

Neglecting the $r_{o}$ caused by channel length modulation, the output impedance $r_{\text {out }}$ is derived as (17) and the transfer function is shown in (18) with the pole and the zero locating at $\mathrm{gm}_{n} / C$ and $1 / R C$, respectively

$$
\begin{aligned}
r_{\mathrm{out}} & =\frac{v_{t}}{i_{t}}=\frac{1+s R C}{g m_{n}+s C} \\
A_{\mathrm{DM}} & =\left|\frac{V_{O-P}-V_{O-N}}{V_{\mathrm{IN}-P}-V_{\mathrm{IN}-N}}\right|=\frac{g m_{p}}{g m_{n}}\left(\frac{1+s R C}{1+s\left(C / g m_{n}\right)}\right) .
\end{aligned}
$$

Consequently, if $R \gg 1 / g m_{n}$, the location of zero is at lower frequencies than that of the pole. Thus, the differential input signal $\left(V_{I N-P}-V_{\mathrm{IN}-\mathrm{N}}\right)$ will have phase lead compared to the differential output signal $\left(V_{O-P}-\mathrm{V}_{O-N}\right)$. The $R$ and $C$ are set as $500 \mathrm{k} \Omega$ and $5 \mathrm{pF}$, respectively, to cause the zero is located about $63.6 \mathrm{kHz}$. The frequency response is depicted in Fig. 7. The compensated zero contributes that the maximum phase delay of $14^{\circ}$ locates at about $200 \mathrm{kHz}$. Consequently, the system is allowed to operate at a lowest frequency $200 \mathrm{kHz}$ without the need of large $R_{\mathrm{ESR}}$. In the meanwhile, the differential structure enhances the noise immunity and decreases the Jitter and the EMI effects. In this paper, the proposed technique ensures the stability although the $200 \mu \mathrm{F}$ MLCC with only $1 \mathrm{~m} \Omega$ ESR is used as the output capacitor.

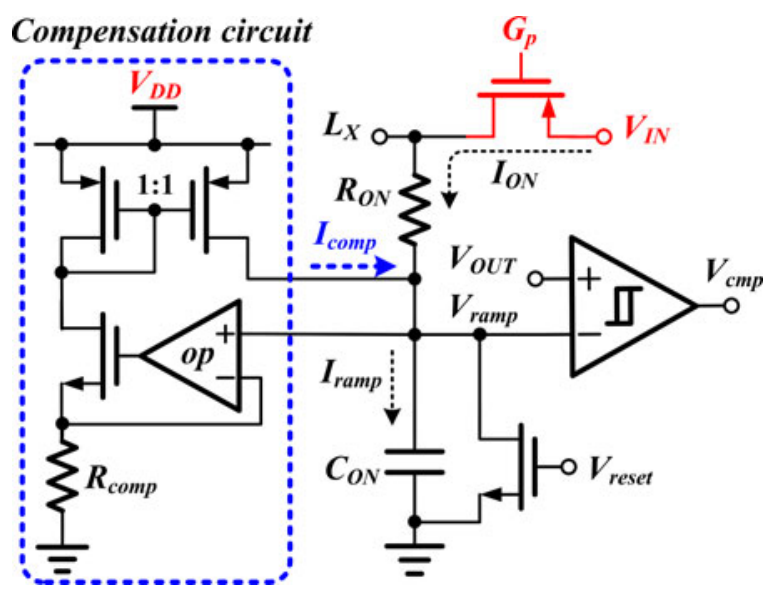

Fig. 15. Proposed AONT circuit for reliable switching frequency without being affected by the input voltage $V_{\mathrm{IN}}$.

\section{B. AONT Circuit}

Without an internal fixed frequency oscillator, an adaptive period of on-time is the solution to compensate the perturbations of $V_{\mathrm{IN}}, V_{\mathrm{OUT}}$, and $I_{\mathrm{LOAD}}$. Therefore, the reliable on-time timer is necessary to avoid frequency variation in steady state and thus stabilize the system without being affected by process, voltage, and temperature (PVT) variations.

The adaptive on-time circuit proposed in [17] derives a nonlinear on-time value if the input voltage changes because of the superfluous parameter from the gate-source voltage $V_{\mathrm{GS}}$ of diode-connected MOSFET. For a wide range of input voltage, the existing inaccuracy caused by the $V_{\mathrm{GS}}$ should be carefully removed. Besides, the ripple-based regulator can also be in accordance with an external clock, either directly or with a phase-locked loop (PLL) [18] and [19]. Unfortunately, it will need additional complicated circuits at the sacrifice of silicon area and cost. Here, Fig. 15 shows the proposed adaptive on-time timer. The charging current $I_{\mathrm{ramp}}$ including both $I_{\mathrm{ON}}$ and $I_{\mathrm{comp}}$ flows into the $C_{\mathrm{ON}}$ during the on-time period and thus the ontime value is independent of the $V_{\mathrm{IN}}$ as expressed in (19) since the $R_{\mathrm{ON}}$ can be equal to the $R_{\mathrm{comp}}$ by good matching methods in chip layout with a little influence of process variations

$$
T_{\mathrm{ON}}=\left(R_{\mathrm{ON}} \cdot C_{\mathrm{ON}}\right) \cdot \frac{V_{\mathrm{OUT}}}{V_{\mathrm{IN}}} .
$$

The (19) shows that the $T_{\mathrm{ON}}$ is simply determined by the $R_{\mathrm{ON}}$ and the $C_{\mathrm{ON}}$ without being affected by the $V_{\mathrm{GS}}$. The desired switching frequency can be obtained by trimming the capacitor $C_{\mathrm{ON}}$.

Substituting (19) into (1), the $f_{\mathrm{SW}}$ can be kept constant, irrespective of the $V_{\mathrm{OUT}}$ and the $V_{\mathrm{IN}}$. Comparing with prior arts, this circuit provides more accurate and linear solution to correctly minimize the variation of frequency. Therefore, the proposed COT control works as the function of the PWM operation even without the internal clock.

Furthermore, connecting the $R_{\mathrm{ON}}$ to the node $L_{X}$ instead of the $V_{\mathrm{IN}}$ directly, the $I_{\mathrm{ON}}$ is only generated at the on-time period. The advantages include that the $V_{\text {ramp }}$ is always lower than the 
TABLE II

PERFORMANCE OF THE COT CONVERTER

\begin{tabular}{||l|c||}
\hline Process & UMC $0.35 \mu \mathrm{m}$ BCD 40V \\
\hline Input voltage $\left(V_{I N}\right)$ & $5 \sim 21 \mathrm{~V}$ \\
\hline Output voltage $\left(V_{O U T}\right)$ & $0.75-3.3 \mathrm{~V}$ \\
\hline Supply voltage for chip $\left(V_{D D}\right)$ & $5 \mathrm{~V}$ \\
\hline Load range $\left(I_{L O A D}\right)$ & $0.1 \mathrm{~A}-8 \mathrm{~A}$ \\
\hline Inductor & $1 \mu \mathrm{H}$ \\
\hline Output Capacitor $(\mathrm{MLCC})$ & $220 \mu \mathrm{F}\left(22 \mu \mathrm{F}^{*} 10\right)$ \\
\hline$R_{E S R}$ & $1 \mathrm{~m} \Omega$ \\
\hline$L_{E S L}$ & $2.6 \mathrm{nH}$ \\
\hline Operation frequency & $100 \mathrm{KHz}-600 \mathrm{KHz}$ \\
\hline Output ripple & $8 \mathrm{mV}-10 \mathrm{mV}$ \\
\hline Maximum efficiency & $91 \%$ \\
\hline
\end{tabular}

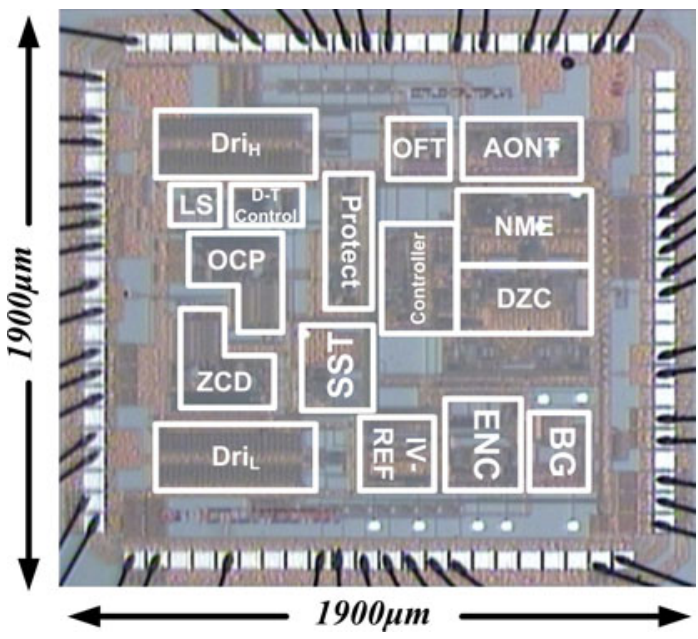

Fig. 16. Chip micrograph

$V_{\text {OUT }}$ and the concern of high-voltage damage caused by the $V_{\text {IN }}$ and wasting power consumption can be avoided. Besides, the number of high-voltage devices can be reduced for low cost due to the proposed method.

\section{EXPERIMENTAL RESULTS}

\section{A. Chip Micrograph}

The COT buck converter with the DZC-NME technique was fabricated in UMC $0.35 \mu \mathrm{m}$ BCD $40 \mathrm{~V}$ process. For application of high-conversion ratio and heavy driving current of $8 \mathrm{~A}$, power MOSFETs are selected as discrete components. The high-side and the low-side power MOSFETs are the AOL1414 and the AOL1412, respectively. The off-chip inductor and the capacitor are selected as $1 \mu \mathrm{H}$ and $220 \mu \mathrm{F}(22 \mu \mathrm{F} \times 10)$, respectively. The specifications of the proposed converter are listed in Table II.

The nominal switching frequency is near $300 \mathrm{kHz}$. The output voltage ranges from 0.75 to $3.3 \mathrm{~V}$ with the input voltage defined by the laptop adapter or the desktop power supply. That is, the highest input voltage can be $21 \mathrm{~V}$. Fig. 16 shows the chip micrograph with the active silicon area about $3.61 \mathrm{~mm}^{2}$ including test circuits. Table III describes the function of the subcircuits.
TABLE III

DESCRIPTION OF SUBCIRCUITS

\begin{tabular}{|c|c|c|c|}
\hline DZC & Differential-Zero Compensator & ZCD & Zero-Current Detector \\
\hline NME & Noise Margin Enhancement & OCP & Over-Current Protector \\
\hline AONT & Adaptive On-Time Timer & PROTECTOR & Protect Circuit \\
\hline OFT & Minimum Off Time Timer & SST & Soft-Start \\
\hline LS & Level Shift & BG & Bandgap \\
\hline D-T Control & Deadtime Control & ENC & Enable Controller \\
\hline Dri $i_{H}$ & High Side Driver & IV-REF & $\begin{array}{c}\text { Biasing Current / Reference } \\
\text { Voltage Generator }\end{array}$ \\
\hline Dri & Low Side Driver & & \\
\hline
\end{tabular}

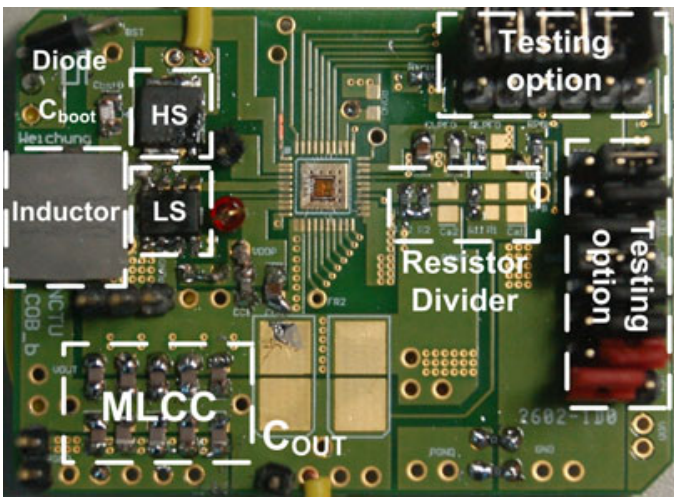

Fig. 17. Prototype of the proposed COT buck converter.

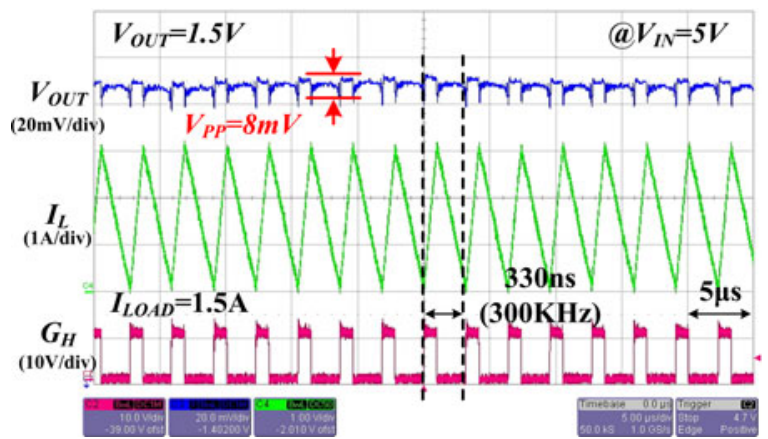

Fig. 18. Waveforms in steady-state with the proposed DZC-NME technique.

Fig. 17 shows the prototype of the COT buck converter with the proposed DZC-NME technique. Here, the MLCC is used as the output capacitor. Simply, the equivalent of the $R_{\mathrm{ESR}}$ value can be calculated as almost $1 \mathrm{~m} \Omega$ only according to the estimated output ripple $V_{\mathrm{pp}}$ and (20) [16]

$$
\begin{aligned}
V_{\mathrm{pp}} & =V_{C_{\mathrm{OUT}}}+V_{\mathrm{ESR}}=\frac{V_{\mathrm{OUT}}(1-D)}{8 f_{\mathrm{SW}}^{2} L C}+\frac{R_{\mathrm{ESR}} V_{\mathrm{OUT}}(1-D)}{f_{\mathrm{SW}} L} \\
& \Rightarrow R_{\mathrm{ESR}}=\left(V_{\mathrm{pp}}-\frac{V_{\mathrm{OUT}}(1-D)}{8 f_{\mathrm{SW}}^{2} L C}\right) \cdot \frac{f_{\mathrm{SW}} L}{V_{\mathrm{OUT}}(1-D)} \cdot(20)
\end{aligned}
$$

\section{B. Steady-State and Load Transient Response}

Fig. 18 shows the steady state of experimental results as the $V_{\mathrm{IN}}$ is $5 \mathrm{~V}$ and the output voltage $V_{\mathrm{OUT}}$ is $1.5 \mathrm{~V}$ when the $I_{\mathrm{LOAD}}$ is $1.5 \mathrm{~A}$ and the switching frequency is $300 \mathrm{kHz}$. The $I_{L}$ is the inductor current. The $G_{H}$ is the driving signal for the high-side MOSFET. Here, the $R_{\mathrm{ESR}}$ is about $1 \mathrm{~m} \Omega$ and the $C_{\mathrm{OUT}}$ is 


\section{Stable with DZC-NME technique}

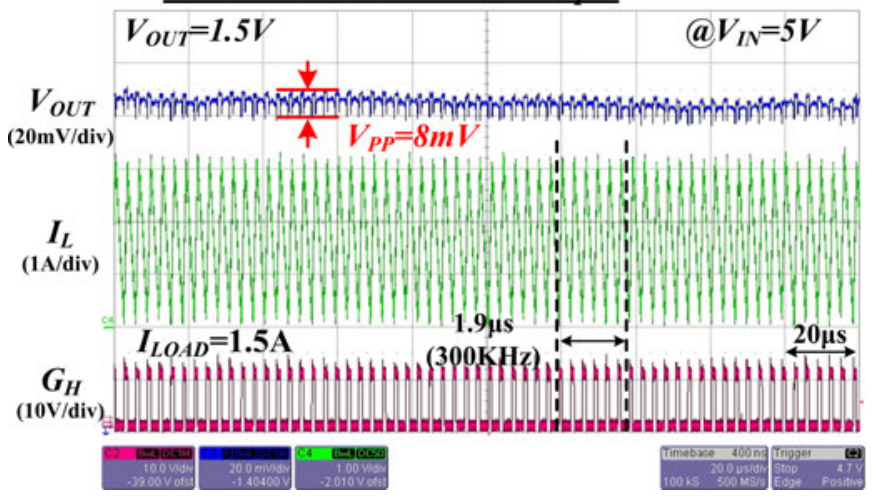

Fig. 19. Regulated waveforms due to the implementation of the DZC-NME technique at $V_{\mathrm{IN}}=5 \mathrm{~V}$ and $V_{\mathrm{OUT}}=1.5 \mathrm{~V}$.

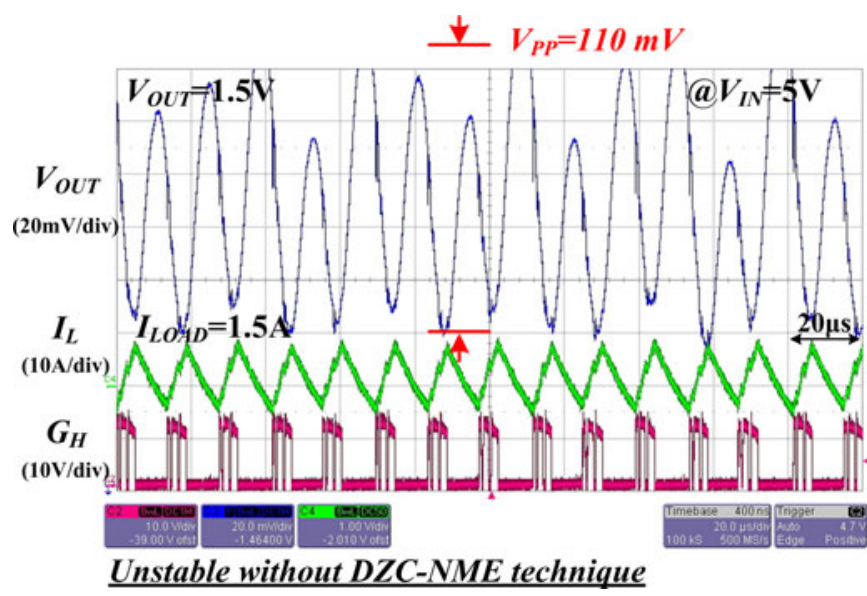

Fig. 20. Unstable waveforms in the COT buck converter without the DZCNME technique at $V_{\mathrm{IN}}=5 \mathrm{~V}$ and $V_{\mathrm{OUT}}=1.5 \mathrm{~V}$.

$220 \mu \mathrm{F}(22 \mu \mathrm{F} \times 10)$ owing to the use of MLCC. According to the conventional stability criteria shown in (12), the switching frequency should be higher than $2 \mathrm{MHz}$. However, experimental results show that the system stability is guaranteed even under the low-switching frequency of $300 \mathrm{kHz}$ since the DZC-NME technique can reduce the limitation of the stability criteria. Thus, the switching loss can be greatly reduced. Specially, the voltage ripple at the $V_{\text {OUT }}$ can be smaller than $8 \mathrm{mV}$ when the $V_{\mathrm{IN}}$ is $5 \mathrm{~V}$. Moreover, the output ripple and the inductor current are out of phase due to the usage of the MLCC.

Figs. 19 and 20 can demonstrate the function of the DZCNME technique when $V_{\text {IN }}$ is $5 \mathrm{~V}$ and $V_{\text {OUT }}$ is $1.5 \mathrm{~V}$. Fig. 19 shows the stable operation due to the implementation of the DZC-NME technique. Contrarily, with external option set by testing circuit, the subharmonic oscillation waveform happens when the DZC-NME is disabled as shown in Fig. 20. The proposed DZC technique contributes the phase lead to the feedback signal and results in the similar performance that utilizes the output capacitor with large ESR.

Furthermore, if the $R_{\mathrm{ESR}}$ is about $1 \mathrm{~m} \Omega$, the effect of ESL is more considerable when the $V_{\mathrm{IN}}$ is higher than $15 \mathrm{~V}$. The $V_{\mathrm{ESL}}$ is $40 \mathrm{mV}$ as shown in Figs. $21-23$. It shows the contribution of the
Stable with DZC-NME technique

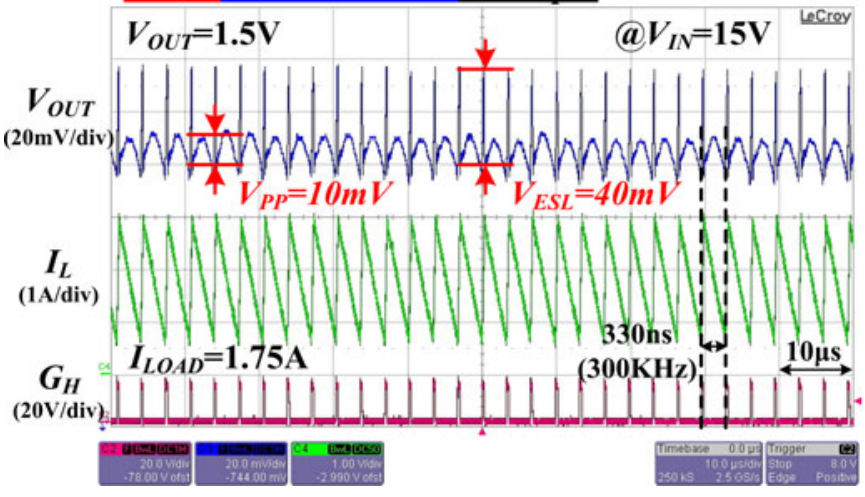

Fig. 21. Regulated waveforms due to the implementation of the DZC-NME technique at $V_{\mathrm{IN}}=15 \mathrm{~V}$ and $V_{\mathrm{OUT}}=1.5 \mathrm{~V}$.

\section{Marginal-Stable with only DZC technique}

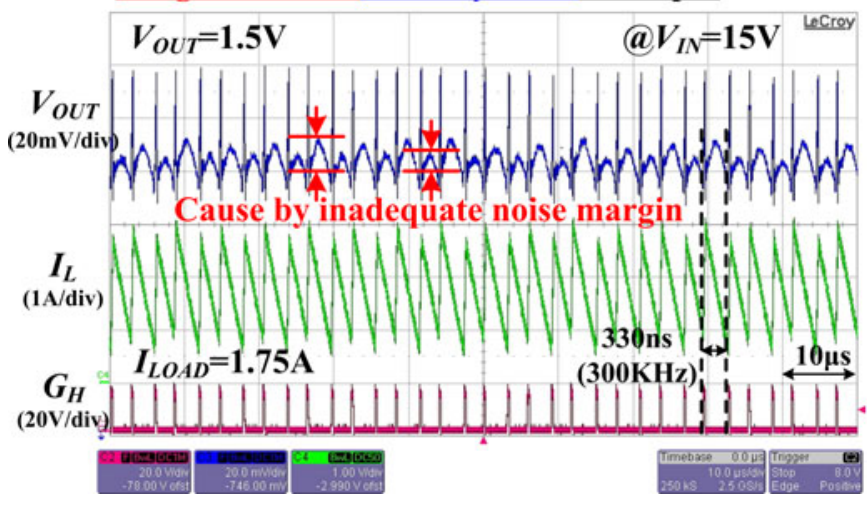

Fig. 22. Marginal stable waveforms with the DZC technique only when the $V_{\mathrm{IN}}$ is $15 \mathrm{~V}$ and the $V_{\mathrm{OUT}}$ is $1.5 \mathrm{~V}$.

\section{Unstable without DZC-NME technique}

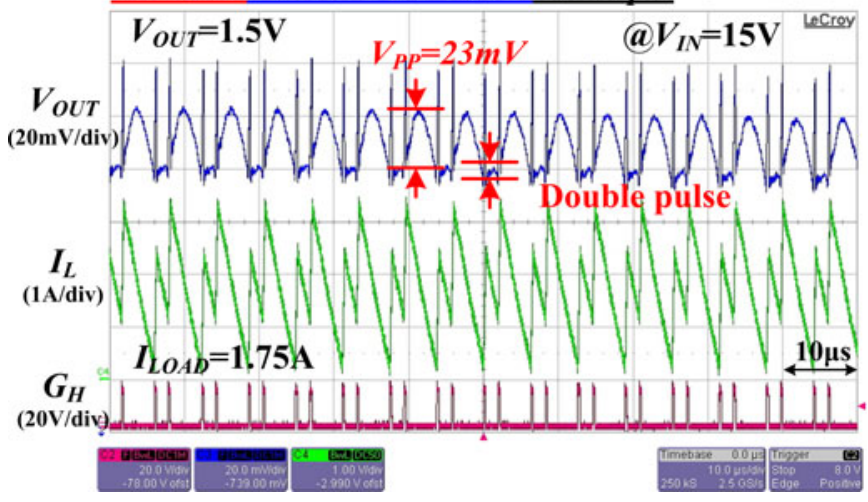

Fig. 23. Unstable waveforms in the COT buck converter without the DZCNME technique when the $V_{\text {IN }}$ is $15 \mathrm{~V}$ and the $V_{\text {OUT }}$ is $1.5 \mathrm{~V}$.

NME circuit if comparing Fig. 21 with Fig. 22. The system with only the DZC circuit has not enough noise margins so that the $V_{\text {OUT }}$ is marginally stable. Fig. 23 shows the seriously unstable waveforms without the aid of the DZC-NME technique.

Fig. 24 shows the waveforms of the $V_{\mathrm{OUT}}$ and the inductor current $I_{L}$ operating at the CCM operation when the load current $I_{\mathrm{LOAD}}$ steps from 1 to $8 \mathrm{~A}$, or vice versa. Here, the $V_{\mathrm{IN}}$ is $15 \mathrm{~V}$ and the $V_{\mathrm{OUT}}$ is $1.5 \mathrm{~V}$. Consequently, the switching frequency is 


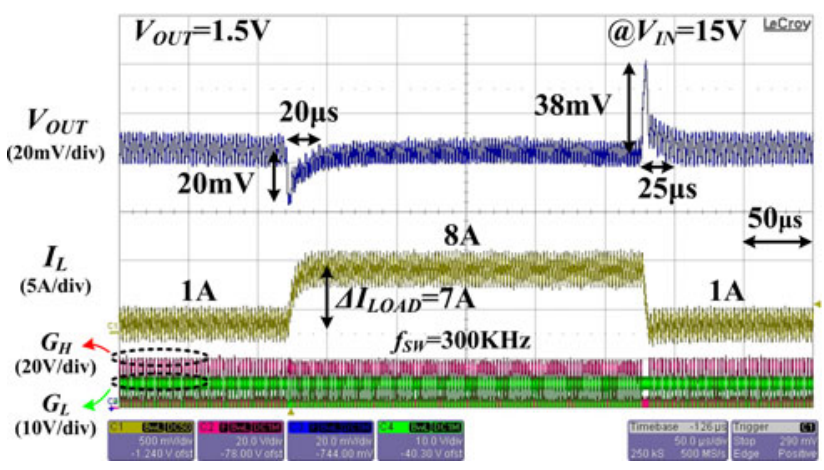

Fig. 24. Load transient response at the CCM operation with the proposed DZC-NME technique.

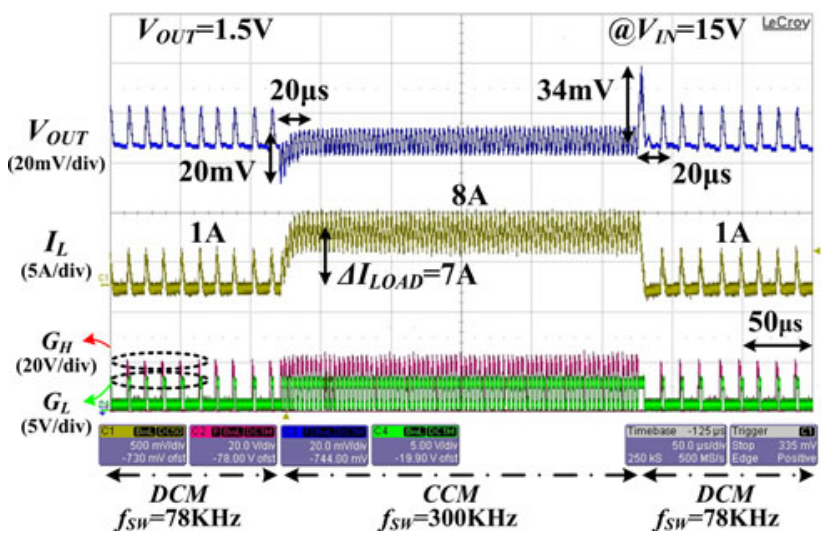

Fig. 25. Load transient response at the DCM operation at light loads with the proposed DZC-NME technique.

TABLE IV

COMPARISONS OF THE PRIOR ARTS

\begin{tabular}{|c|c|c|c|}
\hline & This work & {$[15]$} & {$[20]$} \\
\hline Technology & $\mathbf{0 . 3 5} \boldsymbol{\mu m}$ & $\mathrm{N} / \mathrm{A}$ & $\mathrm{N} / \mathrm{A}$ \\
\hline Controller Methodology & Constant on-time & Constant on-time & $\mathrm{V}^{2} \mathrm{C}$ control \\
\hline Input Voltage $\left(V_{I N}\right)$ & $\mathbf{1 5 V}$ & $25 \mathrm{~V}$ & $12 \mathrm{~V}$ \\
\hline Output Voltage $\left(V_{O U T}\right)$ & $\mathbf{1 . 5 V}$ & $1.05 \mathrm{~V}$ & $5 \mathrm{~V}$ \\
\hline Inductor $\left(L^{\prime}\right)$ & $\mathbf{1 \mu H}$ & $\mathrm{N} / \mathrm{A}$ & $8.6 \mu \mathrm{H}$ \\
\hline Capacitor $\left(C_{O}\right)$ & $\mathbf{2 2 0 \mu} \mathbf{F}$ & $220 \mu \mathrm{F}$ & $4880 \mu \mathrm{F}$ \\
\hline Switching Frequency $\left(f_{S W}\right)$ & $\mathbf{3 0 0} \mathbf{~ K H z}$ & $300 \mathrm{KHz}$ & $95.3 \mathrm{KHz}$ \\
\hline equivalent series resistance $\left(\mathrm{R}_{\mathrm{ESR}}\right)$ & $\mathbf{1 m \Omega}$ & $1 \mathrm{~m} \Omega$ & $12 \mathrm{~m} \Omega$ \\
\hline Output Voltage Ripple $\left(\Delta V_{P P}\right)$ & $\mathbf{1 0 m V}$ & $17 \mathrm{mV}$ & $50 \mathrm{mV}$ \\
\hline Highest Efficiency & $\mathbf{9 1 \%}$ & $\mathrm{N} / \mathrm{A}$ & $\mathrm{N} / \mathrm{A}$ \\
\hline Load transient $\left(\Delta_{L O O A D}\right)$ & $\mathbf{7 A}$ & $8 \mathrm{~A}$ & $14.2 \mathrm{~A}$ \\
& $\mathbf{( 1 A \rightarrow 8 A})$ & $(1 \mathrm{~A} \rightarrow 9 \mathrm{~A})$ & $(5 \mathrm{~A}-19.2 \mathrm{~A})$ \\
\hline Recovery Time $\left(T_{R}\right)$ & $\mathbf{2 5 \mu \mathbf { s }}$ & $30 \mu \mathrm{s}$ & $80 \mu \mathrm{s}$ \\
\hline
\end{tabular}

$300 \mathrm{kHz}$. The undershoot voltage and the overshoot voltage are 20 and $38 \mathrm{mV}$, respectively. The transient recovery time is 20 and $25 \mu$ s, respectively. Fig. 25 shows the waveforms operating at DCM at light loads. The switching frequency $f_{\mathrm{SW}}$ is scaled down to $78 \mathrm{kHz}$ to enhance efficiency. It is the advantage of the COT control for high efficiency at light loads. Obviously, the system stability can be guaranteed when the output operates under different load condition owing to the implementation of the DZC-NME technique.

\section{Comparison With Other Techniques}

Table IV lists the comparison result with the prior arts in the design of COT control dc-dc converters. Obviously, the output

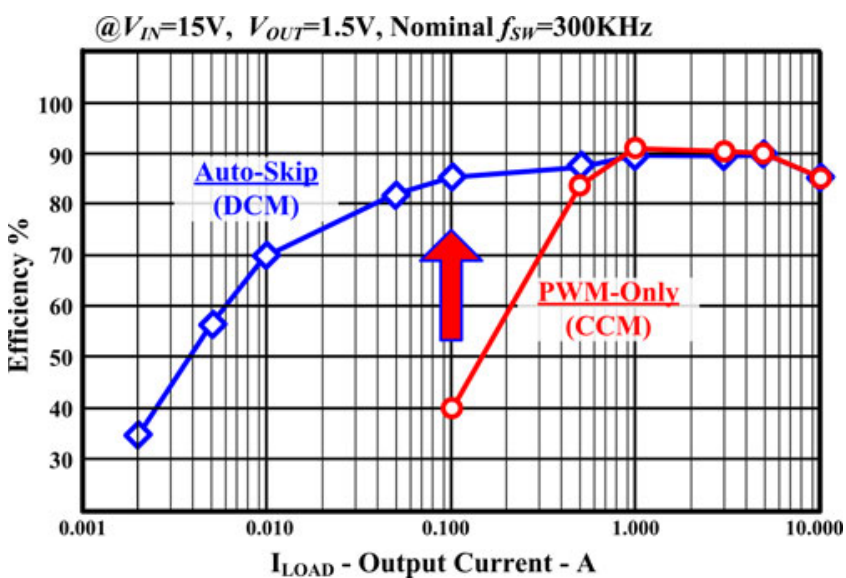

Fig. 26. Power conversion efficiency of the dc-dc converter with the proposed DZC-NME technique.

ripple has been effectively reduced because of using MLCC. The system can also operate at a lower switching frequency when using small ESR. It can demonstrate the high performance achieved by the DZC-NME technique.

\section{Power Conversion Efficiency and Power Consumption}

Fig. 26 shows the power conversion efficiency of the proposed dc-dc converter with the DZC-NME technique. The efficiency can be kept at $91 \%$ at medium to heavy loads. The light-load efficiency is deteriorated since the switching loss dominates the total power consumption. However, the efficiency at light loads can be improved by COT control when the converter enables the ZCD circuit and operates at the Auto-Skip mode compared to the converter simply operates the normal PWMonly mode. The proposed buck converter not only works at nearly constant frequency similar to PWM operation but also decreases the switching frequency according to the loading like the PFM operation for high efficiency at light loads.

\section{CONCLUSION}

The DZC-NME technique is proposed in this paper to conquer the small ESR value and large ESL effect in the COT buck converter. Even though the MLCC is used as the output capacitor if without conventional ESR compensation, the DZC technique still can increase the system stability since the compensator contributes phase lead similar to the PD controller. Besides, the differential structure can benefit the noise margin to decrease the Jitter and the EMI effects. On the other hand, the NME technique eliminates the effect of ESL to enhance the noise immunity. Furthermore, using the reliable on-time timer with an improved linear function, the near-constant switching frequency, which is adjusted to accommodate to variable input voltage, can further confirm the system stability. Because of MLCC with extremely small $R_{\mathrm{ESR}}$ value for general applications, the output ripple can be greatly reduced and thus switching power loss can be decreased in corresponding to large $R_{\mathrm{ESR}}$ used to compensate conventional ripple-based control. Experiment results verify the correct and effective functions of the DZC and the NME 
techniques at the strict case when small $R_{\mathrm{ESR}}$ of $1 \mathrm{~m} \Omega$ and large $V_{\mathrm{ESL}}$ of $40 \mathrm{mV}$. Without scarifying the inherent advantages of the COT control, the DZC-NME technique for the MLCC applications can ensure low ripple of $10 \mathrm{mV}$ and high efficiency of $91 \%$.

\section{REFERENCES}

[1] C.-Y. Hsieh and K.-H. Chen, "Adaptive pole-zero position (APZP) technique of regulated power supply for improving SNR," IEEE Trans. Power Electron., vol. 23, no. 6, pp. 2949-2963, Nov. 2008.

[2] W.-R. Liou, M.-L. Yeh, and Y. L. Kuo, "A high efficiency dual-mode buck converter IC for portable applications," IEEE Trans. Power Electron., vol. 23, no. 2, pp. 667-677, Mar. 2008.

[3] P.-J. Liu, Y.-K. Lo, H.-J. Chiu, and Y.-J. E. Chen, "Dual-current pump module for transient improvement of step-down DC-DC converters," IEEE Trans. Power Electron., vol. 24, no. 4, pp. 985-990, Apr. 2009.

[4] R. P. Singh and A. M. Khambadkone, "A buck-derived topology with improved step-down transient performance," IEEE Trans. Power Electron., vol. 23, no. 6, pp. 2855-2866, Nov. 2008.

[5] M.-H. Huang and K.-H. Chen, "Single-inductor multi-output (SIMO) DC-DC converters with high light-load efficiency and minimized crossregulation for portable devices," IEEE J. Solid-State Circuits, vol. 44, no. 4, pp. 1099-1111, Apr. 2009.

[6] H.-H. Huang, C.-L. Chen, D.-R. Wu, and K.-H. Chen, "Solid-duty-control technique for alleviating the right-half-plane zero effect in continuous conduction mode boost converters," IEEE Trans. Power Electron., vol. 27, no. 1, pp. 354-361, Jan. 2012.

[7] Y.-H. Lee, S.-C. Huang, S.-W. Wang, W.-C. Wu, P.-C. Huang, H.-H. Ho, Y.-T. Lai, and K.-H. Chen, "Power-tracking embedded buck-boost converter with fast dynamic voltage scaling for the SoC system," IEEE Trans. Power Electron., vol. 27, no. 3, pp. 1271-1282, Mar. 2012.

[8] M.-H. Huang, P.-C. Fan, and K.-H. Chen, "Low-ripple and dual-phase charge pump circuit regulated by switched-capacitor-based bandgap reference," IEEE Trans. Power Electron., vol. 24, no. 5, pp. 1161-1172, May 2009

[9] F. L. Luo and H. Ye, "Analysis and modeling of on-chip charge pump design based on pumping gain increase circuits with a resistive load," IEEE Trans. Power Electron., vol. 23, no. 4, pp. 2187-2194, Jul. 2008.

[10] S. Qu, "Modeling and design considerations of $\mathrm{V}^{2}$ controlled buck regulator," in Proc. IEEE Appl. Power Electron. Conf., Mar. 2001, vol. 1, pp. 507-513.

[11] F. Wang, J. Xu, and J. Xu, "Small-signal model of $\mathrm{V}^{2}$ control technique with compensation," in Proc. IEEE Int. Conf. Commun., Circuits Syst., Jun. 2004, vol. 2, pp. 27-29.

[12] J. Sun, "Characterization and performance comparison of ripple-based control methods for voltage regulator modules," IEEE Trans. Power Electron., vol. 21, no. 2, pp. 346-353, Mar. 2006.

[13] R. Redl and J. Sun, "Ripple-based control of switching regulators, an overview," IEEE Trans. Power Electron., vol. 24, no. 12, pp. 2669-2680, Dec. 2009.

[14] Y.-H. Lee, S.-J. Wang, and K.-H. Chen, "Quadratic differential and integration technique in $\mathrm{V}^{2}$ control buck converter with small ESR capacitor," IEEE Trans. Power Electron., vol. 25, no. 4, pp. 829-838, Apr. 2010.

[15] C.-J. Chen, D. Chen, C.-W. Tseng, C.-T. Tseng, Y.-W. Chang, and K.-C. Wang, "A novel ripple-based constant on-time control with virtual inductor current ripple for Buck converter with ceramic output capacitors," in Proc. IEEE Appl. Power Electron. Conf., Mar. 2011, pp. 1488-1493.

[16] R. W. Erickson and D. Maksimovic, Fundamentals of Power Electronics, 2nd ed. Norwell, MA: Kluwer, 2001.

[17] B. Sahu and G. A. Rincon-Mora, "An accurate, low-voltage, CMOS switching power supply with adaptive on-time pulse-frequency modulation (PFM) control," IEEE Trans. Circuits Syst. I, Reg. Papers, vol. 54, no. 2, pp. 312-321, Feb. 2007.

[18] C.-H. Tso and J.-C. Wu, "A ripple control buck regulator with fixed output frequency," IEEE Power Electron. Lett., vol. 1, no. 3, pp. 61-63, Sep. 2003.

[19] K. Lee, F. C. Lee, and M. Xu, "Novel hysteretic control method for multiphase voltage regulators," in Proc. IEEE Appl. Power Electron. Conf., Feb. 2008, pp. 1508-1514

[20] F. Wang, J. Xu, and B. Wang, "Comparison study of switching DC-DC converter control techniques," in Proc. IEEE Int. Conf. Commun., Circuits Syst., Jun. 2006, vol. 4, pp. 2713-2717.

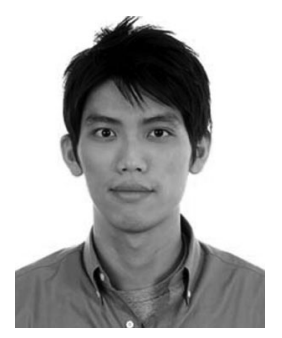

Wei-Chung Chen (S'12) was born in Yunlin, Taiwan. He received the B.S. degree from the Department of Electrical Engineering, National Sun Yat-sen University, Kaohsiung, Taiwan, in 2010, and the M.S. degree from National Chiao Tung University, Hsinchu, Taiwan, in 2012, where he is currently working toward the Ph.D. degree at the Institute of Electrical and Computer Engineering.

$\mathrm{He}$ is a Member of the Mixed-Signal and Power Management IC Laboratory, Institute of Electrical and Computer Engineering, National Chiao Tung University. His research interests include the power management IC design, analog integrated circuits, and mixed signal IC design.

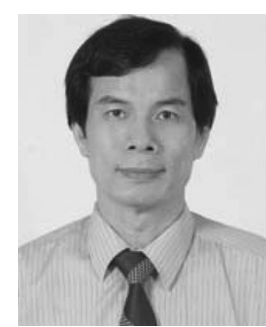

Ching-Sung Wang received the M.S. degree in electronic engineering from the Chung Yuan Christian University, Taoyuan, Taiwan, in 1987, and the Ph.D. degree in electrical engineering from the National Taiwan University, Taipei, Taiwan, in 1998.

$\mathrm{He}$ is currently an Associated Professor in the Department of Electronic Engineering, Oriental Institute of Technology, Taipei. He is also the Director of Health-Care Technology and Research Center at the same institute. His current interests include to develop health-care information system, biomedical instrumentation, power management ICs, and mixed-signal circuit designs.

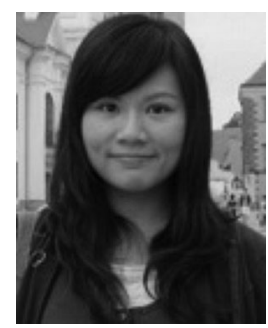

Yi-Ping Su was born in Taipei, Taiwan. She received the B.S. degree from the Department of Electrical Engineering, National Sun Yat-sen University, Kaohsiung, Taiwan, in 2009. She is currently working toward the $\mathrm{Ph} . \mathrm{D}$. degree at the Institute of Electrical Engineering, National Chiao Tung University, Hsinchu, Taiwan.

She is also a Faculty Member at the Mixed Signal and Power Management IC Laboratory, Institute of Electrical Engineering. Her current research interests include the power management integrated circuits design, battery charging integrated circuits design, and analog integrated circuits design.

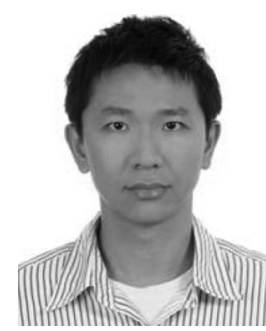

Yu-Huei Lee (S'09) was born in Taipei, Taiwan. He received the B.S. and M.S. degrees from the Department of Electrical and Control Engineering, National Chiao Tung University, Hsinchu, Taiwan, in 2007 and 2009 , respectively, where he is currently working toward the Ph.D. degree at the Institute of Electrical Control Engineering.

$\mathrm{He}$ is also a Faculty Member at the Mixed Signal and Power IC Laboratory, Institute of Electrical Control Engineering. His current research interests include the power management integrated circuit design, light-emitting diode driver IC design, and analog integrated circuits. 


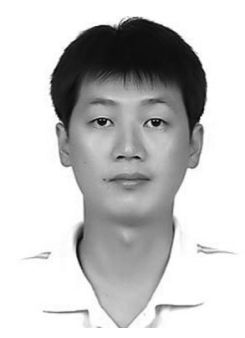

Chia-Ching Lin was born in Taoyuan, Taiwan. He received the B.S. degree from the Department of Electrical Engineering, Southern Taiwan University of Science and Technology, Tainan, Taiwan, in 2002, and the M.S. degree from National Chiao Tung University, Hsinchu, Taiwan, in 2011.

From 2002 to 2005, he was an Analog Circuit Designer with Glovic, Inc., Hsinchu, Taiwan. From 2005 to 2011, he was engaged in mixed-signal design at Princeton, Inc., Hsinchu. He is currently an R\&D Engineer at ILT Technology Corp., Hsinchu, Taiwan. His research interests include mixed-signal circuit and power management integrated circuit designs.

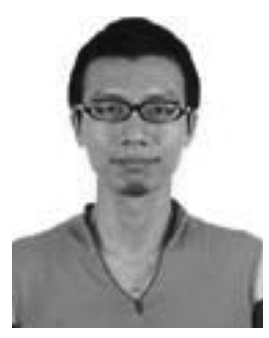

Ming-Jhe Du was born in Tainan, Taiwan, in 1981. $\mathrm{He}$ received the B.S. degree in electrical engineering from National Cheng Kung University, Tainan, Taiwan, in 2003. He received the M.S. degree in electrical engineering from Nation Taiwan University, Taipei, Taiwan, in 2006.

From June 2006 to-date, he is an R\&D Engineer at Industrial Technology Research Institute, Hsinchu, Taiwan.

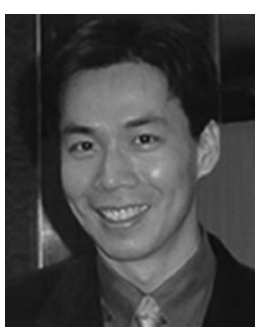

Ke-Horng Chen (M'04-SM'09) received the B.S., M.S., and Ph.D. degrees in electrical engineering from National Taiwan University, Taipei, Taiwan, in 1994, 1996, and 2003, respectively.

From 1996 to 1998, he was a part-time IC Designer at Philips, Taipei. From 1998 to 2000, he was an Application Engineer at Avanti, Ltd., Taiwan. From 2000 to 2003, he was a Project Manager at ACARD, Ltd., where he was engaged in designing power management ICs. He is currently a Professor in the Department of Electrical Engineering, National Chiao Tung University, Hsinchu, Taiwan, where he organized a Mixed-Signal and Power Management IC Laboratory. He is the author or coauthor of more than 80 papers published in journals and conferences, and also holds several patents. His current research interests include power management ICs, mixed-signal circuit designs, display algorithm and driver designs of liquid crystal display TV, red, green, and blue color sequential backlight designs for optically compensated bend panels, and low-voltage circuit designs.

Dr. Chen has served as an Associate Editors of the IEEE TRANSACTIONS ON POWER ELECTRONICS and the IEEE TRANSACTIONS ON CIRCUITS AND SYSTEMS II. He is on the IEEE Circuits and Systems (CAS) VLSI Systems and Applications Technical Committee, and the IEEE CAS Power and Energy Circuits and Systems Technical Committee. 